\title{
On-chip fabrication and in-flow 3D-printing of cell- laden microgel constructs: From chip to scaffold materials in one integral process
}

Benjamin Reineke ${ }^{1,2}$, Ilona Paulus ${ }^{3}$, Jonas Hazur ${ }^{6}$, Madita Vollmer ${ }^{4}$, Gültekin Tamgüney ${ }^{4,5}$, Stephan Hauschild ${ }^{1}$, Aldo R. Boccacini ${ }^{6}$, Jürgen Groll ${ }^{3}$, Stephan Förster ${ }^{* 1,2}$

${ }^{1}$ Jülich Centre for Neutron Science (JCNS-1/IBI-8), Forschungszentrum Jülich GmbH, 52425 Jülich, Germany

${ }^{2}$ Institute of Physical Chemistry, RWTH Aachen University, 52074 Aachen, Germany

${ }^{3}$ Department of Functional Materials in Medicine and Dentistry (FMZ) and Bavarian Polymer Institute (BPI), University of Würzburg, 97070 Würzburg, Germany

${ }^{4}$ Forschungszentrum Jülich GmbH, Institute of Biological Information Processing - Structural Biochemistry (IBI7), Jülich, Germany

${ }^{5}$ Heinrich-Heine-Universität Düsseldorf, Institut für Physikalische Biologie, Düsseldorf, Germany

${ }^{6}$ Institute of Biomaterials, University of Erlangen-Nuremberg, Cauerstr. 6, 91058, Erlangen, Germany

*Corresponding author: s.foerster@fz-juelich.de

Bioprinting has evolved into a thriving technology for the fabrication of cell-laden scaffolds. Bioinks are the most critical component for bioprinting. Recently, microgels have been introduced as a very promising bioink enabling cell protection and the control of the cellular microenvironment. However, their microfluidic fabrication inherently seemed to be a limitation. Here we introduce a direct coupling of microfluidics and 3D-printing for the microfluidic production of cell-laden microgels with direct in-flow bioprinting into stable scaffolds. The methodology enables the continuous on-chip encapsulation of cells into monodisperse microdroplets with subsequent in-flow cross-linking to produce cell-laden microgels, which after exiting a microtubing are automatically jammed into thin continuous microgel filaments. The integration into a 3D printhead allows direct in-flow printing of the filaments into free-standing three-dimensional scaffolds. The method is demonstrated for different cross-linking methods and cell lines. With this advancement, microfluidics is no longer a bottleneck for biofabrication. 
Bioprinting is an emerging technology for the fabrication of three-dimensional cell-laden structures to mimic or replace body tissue. (1) It plays an important role for tissue engineering and drug delivery, as well as for the study of diseases and the development of treatments. In bioprinting, cells and materials are formulated to a bioink $(2,3)$ which is then directly printed into a hierarchically structured 3D scaffold. The ultimate aim of bioprinting is to produce cell-laden scaffolds that exhibit the full or partial function of the target tissue or organ, based on the rationale that the three dimensionally fabricated structural arrangement facilitates and accelerated biological maturation. (4)

Bioinks are thus a crucial component of the bioprinting technology. They rely on materials, mostly hydrogels, which can encapsulate cells and bioactive molecules, and can be used in suited printing technologies such as extrusion printing to fabricate the desired three-dimensional scaffolds or constructs. The design of bioinks is challenging, because their flow and elastic properties need to be finely adjusted to be sufficiently shear-thinning during extrusion, and subsequently developing the desired mechanical stability and elasticity quickly after solidification to form a stable scaffold. Furthermore, bioinks must be biocompatible, sufficiently biofunctional to promote cell viability and proper post-fabrication behavior, and be well perfusable for sufficient provision of nutrients and oxygen.

As bioinks most prominently hydrogel precursor solutions are used, sometimes in a slightly precrosslinked form, and cross-linked after printing to stabilize the constructs. Challenges that often occur are cell sedimentation, mixing inhomogeneities and a trade off between biocompatible formulation and printing and the resulting shape fidelity, since the cells are directly experiencing shear forces in the flowing formulation.

In order to overcome these limitations, Highley et al. (5) recently proposed the use of microgel bioinks. Due to their colloidal nature, microgel bioinks are well shear-thinning and rapidly solidify under quiescent conditions, while cells loaded into the soft colloids are shear protected. Printed microgel scaffolds can, in the case that interfacial polymer entanglement is not sufficient, be further stabilized by secondary crosslinking. Microgels further offer the advantage to tailor the cell microenvironment. Therefore, methods to prepare cell-laden microgels have already been developed, particularly in the area of microfluidics where microgels with very uniform size can be fabricated in a continuous process. (6-8) It was demonstrated that microgels can be formed by physical cross-linking such as via alginate/ $\mathrm{Ca}^{2+}$ ionic complex formation $(9,10)$ which can be modulated by EDTA-complexation $(11,12)$, or by thermally induced gelation such as via cooling gelatin solutions below $20^{\circ} \mathrm{C}$. $(9,13)$ Chemical cross-linking reactions offer greater stability and better mechanical properties of the microgels. Examples are Michael type reactions as for functionalized gelatins, hyalunorates, polyethylene glycols or polyglycerols (12, 14-16), azide-alkyne click reactions as for polyglycerols (17) and photo-crosslinking (18) which requires a photoinitiator and 
crosslinking groups as has been shown for polyethylene glycol. Cells that have been encapsulated include stem cells (9,12,14,15), Crypt and Peyer cells (10), liver cells (HepG2) and endothelial cells (HUVEC) (18), as well as NIH 3T3 fibroblasts (6). The fabrication of functional scaffolds based on cell-laden microgels has so far been shown by Fan et al. (19) using emulsion-based fabrication of gel-MA microgels and by Compaan et al. (20) for gelatin microgel filler particles. For microfluidic generated microgels this has recently been demonstrated for the first time by Highley et al. (5).

The reason for the so far limited number of studies on microgel based bioinks and their bioprinting to scaffolds is the required combination of microfluidics that produces only small microgel quantities, followed by a number of post-chip batch process steps involving cross-linking, the preparation of the bioink, and the subsequent bioprinting into the desired scaffold. This currently makes microgel biofabrication a time-consuming, low-productivity multistep process.

It would therefore be highly desirable if microfluidics for the production of the microgels and bioprinting for the fabrication of the desired scaffolds could be integrated into one continuous, automatable process. Here we show, that microfluidic chips can be designed for the on-chip encapsulation of cells into droplets, which are photo-crosslinked in-flow to produce microgels, which are then automatically jammed from a downstream microtubing to continuously form thin microgel filaments. The microtubing is integrated into the printhead of a 3D-printer to directly in-flow print the filaments into free-standing three-dimensional scaffolds within a prefabricated permeation chamber. We demonstrate that the printed cell-laden microgel scaffolds have sufficient intrinsic microporosity for continuous permeation of medium, and demonstrate the integration of additional complementary perfusable microchannel systems connected to the in/outflow ports of the permeation chamber.

\section{Results and discussion}

\section{Microfluidic device and controlled droplet production}

Our aim was to design a microfluidic chip for (i) low shear stress cell encapsulation, (ii) variability for physical or chemical cross-linking, (iii) a large variation of microdroplet diameter, and (iv) capability to couple it to a 3D-printer. The design should therefore minimize shear forces on cells within narrow channel sections for high cell viability. To allow performing different physical and chemical crosslinking reactions, the entrance channel design should allow sequential mixing of the solutions containing cells, polymers, cross-linking and further agents. The microdroplets should have controllable diameters from $300 \mu \mathrm{m}$ down to $50 \mu \mathrm{m}$ to reach cell-densities of $10^{6} / \mathrm{ml}$ if single cell encapsulation would be desired. 
a)

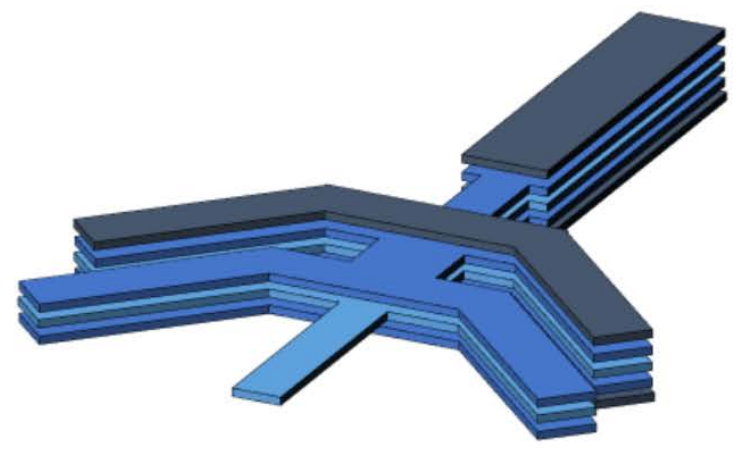

b)

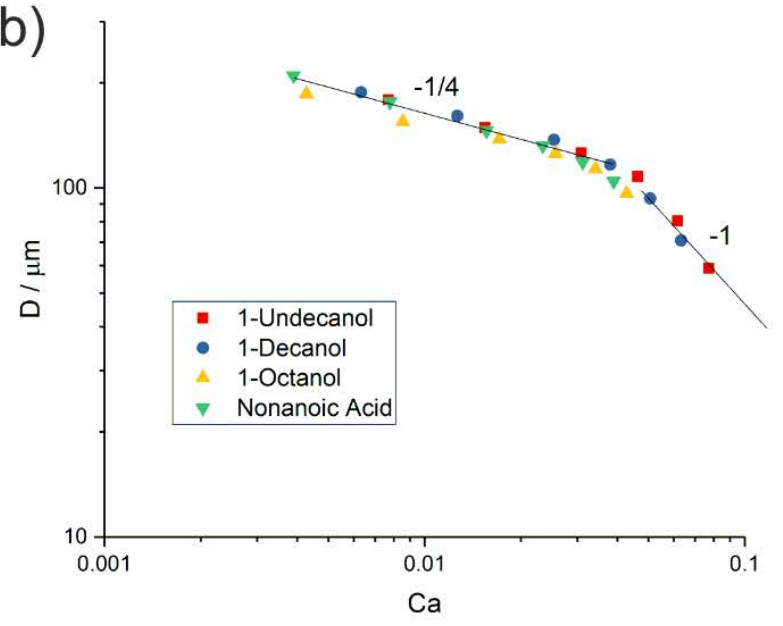

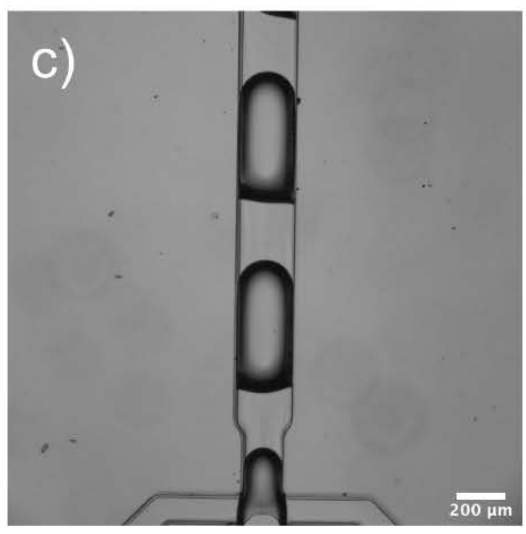
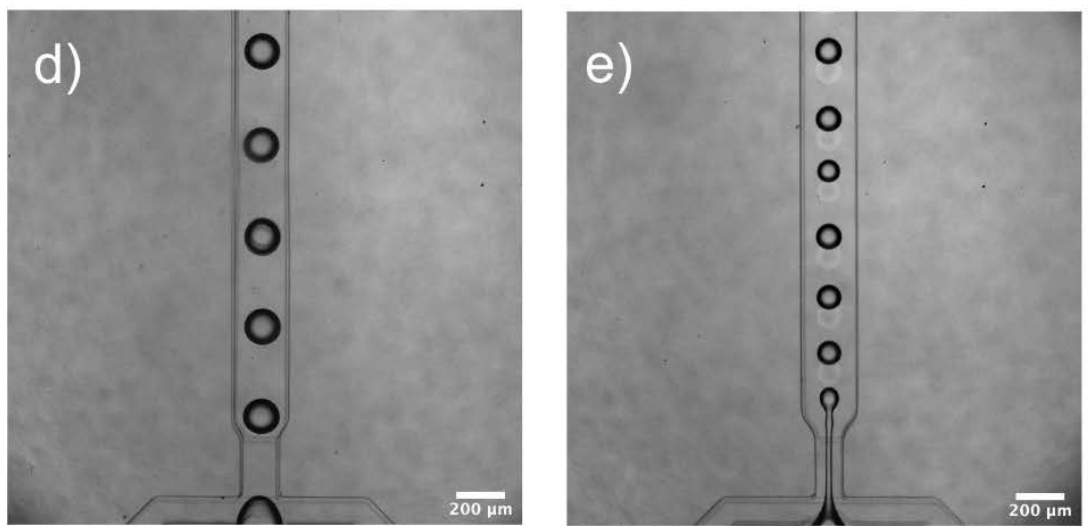

Fig. 1: Three-dimensional schematic view of the multilayer double 3D-focusing microfluidic channel system, (b) control of droplet diameter via the Capiilary number $\mathrm{Ca}$, and accessible hydrodynamic regimes for droplet production: squeezing (c), dripping (d) and jetting (e). The scale bars are $200 \mu \mathrm{m}$.

We therefore used a channel design that allows three-dimensional flow-focusing with two subsequent mixing crosses, followed by a downstream narrow orifice for controlled droplet formation. The design is schematically shown in Fig. 1. It contains an inlet channel for the center stream solution containing the cells and the precursor polymers, and two side channels which can contain buffer solutions, culture media, bioactive substances or crosslinking agents. The side channel streams focus the inlet channel stream threedimensionally into the center of the channel where shear-forces on the cells are minimal. Subsequently, the aqueous stream is three-dimensionally focused with the oil phase to enter a narrow orifice section that controls droplet formation. The narrow section enables to access different hydrodynamic regimes to vary the droplet size over a wide range. The downstream channel is kept sufficiently narrow such that the droplets form a stable droplet train on the channel center streamline. The 3D double focusing chips were 
fabricated by soft lithography using a multilayer technique, and the flows simulated as described in the Supporting Information (Figs. S2-S4, S7).

Droplet break-up occurs when the viscous shear force $F_{\text {shear }}$ exerted by the outer fluid exceeds the pinning interfacial force $F_{\gamma}$ arising from the surface tension. Both forces are directly related by the dimensionless Capillary number $\mathrm{Ca}=F_{\text {shear }} / F_{\gamma}$, which can be calculated in terms of the viscosity of the continuous oil phase $\eta$, the average inlet flow velocity $v$, and the surface tension $\gamma$ as $\mathrm{Ca}=\frac{F_{\text {shear }}}{F_{\gamma}}=\frac{\eta v}{\gamma}$. Depending on the Capillary number, different relevan hydrodynamic regimes for droplet generation can be distinguished: (a) squeezing regime with channel geometry-controlled break-up (Ca < 0.1), (b) dripping regime $(0.1<\mathrm{Ca}<1)$ and $(\mathrm{c})$ jetting regime $(\mathrm{Ca}>1)$. (21-25) As shown in Fig. 1, with the variable 3Dconstriction design all three hydrodynamic regimes for droplet production are accessible, and the Capillary number is the main control parameter for droplet production. It allows to precisely control the droplet diameter in the target range between 50 to $300 \mu \mathrm{m}$ by adjusting the volumetric flow rate, oil viscosity and interfacial tension. The respective viscosities and interfacial tensions are summarized in Table SI in the Supporting Information.

\section{Cell encapsulation}

The cells were encapsulated into the droplets by dispersing them at a given concentration in the center stream (see Fig. 1). The flow-focusing into the center streams at the two mixing crosses is shown in the Supporting Information (Fig. S8). For the tests we used yeast cells (saccharomyces cerevisiae) that were fluorescently labelled with Nile Red. To prevent sedimentation in the syringes, we used OptiPrep solution (16 wt\%). We further used Yellow fluorescent protein (YFP)-tagged HEK293T cells. The number of encapsulated cells per droplet follows a Poisson distribution, as shown in the Supplementary Information (Fig. S18). The microgels were fluorescently labelled by the incorporation with FITC-dextrane which was added in the center stream $\mathrm{A}$.

\section{In-flow photo-crosslinking}

To enable continuous on-chip crosslinking, the crosslinking reaction times should be in the millisecond regime such that for routine flow velocities of $v=2-10 \mathrm{~mm} / \mathrm{s}$ full conversion is reached during the residence time of the microdroplets in the respective microfluidic channel section. It has been previously shown that UV-crosslinking of polymer precursors within microdroplets is a fast and efficient method to produce microgels of controlled size and shape. (26-28) We employed an LED operating at an emission 
wavelength of $\lambda=385 \mathrm{~nm}$ suitable for the efficient use of the water-soluble photoinitiator lithium phenyl2,4,6-trimethylbenzoylphosphinate (LAP). The LED has an illumination power of $P=530 \mathrm{~mW}$ from a 1 $\mathrm{mm}^{2}$ source area. The LED power can be varied to reach high conversion at minimal illumination time to minimize potential cell damage. For a standard flow velocity of $v=10 \mathrm{~mm} / \mathrm{s}$, the residence times over an illuminated distance of $1 \mathrm{~mm}$ is $\mathrm{t}=100 \mathrm{~ms}$. Thus, during this time the droplet is illuminated with an energy of up to $E=P \cdot t=5.3 \mathrm{~J} / \mathrm{cm}^{2}$. This is more than 10-times higher than an energy density of $E=0.45 \mathrm{~J} / \mathrm{cm}^{2}$ reported for complete photo-crosslinking of microgels using a power density of $P=15 \mathrm{~mW} / \mathrm{cm}^{2}$ over a time period of $30 \mathrm{~s}$ (5). Thus, we expected to reach full conversion for continuous in-flow crosslinking using LAP as a photoinitiator.

In our first device versions the LED was positioned directly above the microfluidic reaction channel. We found that the on-chip LED illumination caused prepolymerization of the polymer precursor in the entrance channel sections due to the strong light scattering of the chip material. Since the Rayleigh scattering intensity depends on the wavelengths as $1 / \lambda^{4}$, short wavelength light shows very high scattering intensity. We therefore performed the in-flow LED photo-crosslinking step in a separate and shielded microtubing section downstream the microfluidic chip as shown in Fig. 2. We found that an issue with having the cross-linking step in the downstream microtubing section is the occurrence of microgel bunching. This leads to the formation of microgel clusters having aperiodic exit times at the microtube exit. This disturbs stable microgel filament formation which is necessary for subsequent 3D-printing, as discussed below.

An important factor causing droplet and microgel bunching is the difference between the microchannel and microtube width. It is particularly critical for the transition section from microchannel to microtube, which often is designed as a $90^{\circ}$-bend where the horizontal microchannel enters the vertical punch hole containing the microtube. If the punchhole or the microtube have larger diameters than the microchannel, radial velocity components in the widening sections move the droplets or microgels to off-center streamlines, particularly in bent sections. As in the widened sections the center streamline, and even more the off-center streamlines, have lower flow-velocities, microgels jam into contact, thereby forming bunches or clusters. This was investigated in more detail using FE-simulations as described in the Supporting Information (Figs. S5, S6, S9). 


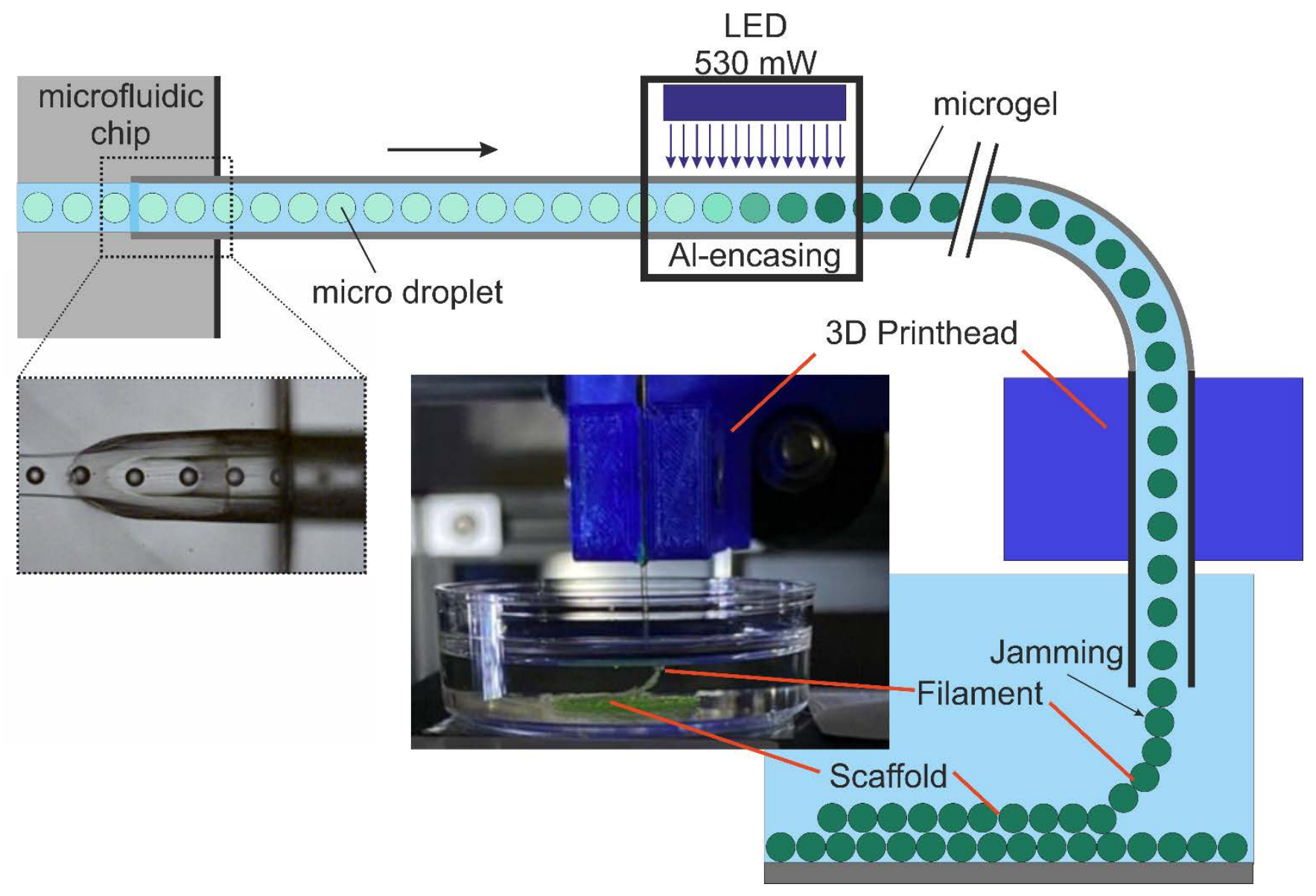

Fig. 2: Scheme of the LED photo-crosslinking and 3D-printing section of the microfluidic/3D-printing device. The droplet train is transferred from the chip microchannel into a microtubing in a straight section with nearly identical inner channel and inner microtubing diameter. Further downstream, the microtubing passes an LED-section for fast photo cross-linking to generate the microgels. This section is contained in an aluminum encasing to avoid premature crosslinking of polymer precursor in upstream channel sections by stray light. Subsequently, the microtubing is integrated into a 3D-printhead, where the microgels are jammed into a filament that is directly 3D-printed into the scaffold.

We therefore transferred the droplet stream from the microfluidic exit channel (250 $\mu$ m diameter) into a microtubing (PE, $280 \mu \mathrm{m}$ inner diameter) having nearly the same inner diameter in a straight co-axial connection as shown in Fig. 2. We observed that upon transfer of the droplet train into the microtubing, the droplets remain well separated and localized in the center stream. With this setup we achieve stable in-flow photo crosslinking of separated droplets for continuous microgel production. 


\section{Direct in-flow pattern bio-printing}

Our aim was to integrate microfluidics with 3D bio-printing into a continuous process. We therefore skipped the bioink fabrication step and directly inserted the microtubing outlet of the microfluidic device into the print head of a commercial 3D-printer used for fused deposition modeling (FDM). FDM is a 3D printing method that is based on the extrusion of a continuous filament of a molten thermoplastic material through a moving print head nozzle to fabricate free-standing three-dimensional constructs. Fused filament deposition is the most widespread printing methodology for additive manufacturing. It has been originally developed to print filaments from thermoplastic materials, and later been extended to print hydrogel filaments for the fabrication of biological tissue constructs.

A first precondition for the direct coupling of microfluidic flows and 3D-printing is that the flow velocity $v_{\mathrm{m}}$ of the microgels is equal to the velocity $v_{\mathrm{p}}$ of the print head. In microfluidics, routine droplet flow velocities are in a range of $v_{\mathrm{m}}=1-50 \mathrm{~mm} / \mathrm{s}$, which notably is in the range of typical FDM print head speeds of $v_{\mathrm{p}}=10-100 \mathrm{~mm} / \mathrm{s}$ or bioprinting nozzle speeds of $1-50 \mathrm{~mm} / \mathrm{s}$, at pressures of $0.5-3 \mathrm{bar}$ (29). Therefore, direct coupling is possible with FDM printing as well as with bioprinting commercial devices.

A second precondition is that filaments are formed after exiting the print head nozzle. We observed that the microgels formed stable filaments upon exiting the microtube. Filament formation is driven by the reduction of the center stream velocity when the fluid streams widens after passing the microtube exit. Concomitantly, the spherical aqueous microgel domains fuse into a continuous cylindrical filament domain, thereby reducing the oil/water interfacial area. This is the reverse of the droplet formation process occurring in the nozzle section of the microfluidic chip, driven by capillary forces. With decreasing center stream velocity the distance between adjacent microgels is reduced until they are jammed into surface contact, thereby forming a pearl-necklace type filament. This is schematically shown in Fig. 2. The filament is stabilized by direct contact adhesion (tack) of the microgels. We attribute the notable adhesion of microgels to chain interpenetration and entanglement formation of the highly mobile chain segments at the periphery of the microgels. This adhesion was already apparent during microgel bunching occurring in earlier versions of the microfluidic channel/tube sections as described above. The freshly formed filament is then deposited at the desired location by the print head. For this, the microtube end is positioned ca. $1 \mathrm{~mm}$ above the substrate or construct surface. We find that standard substrates such as polystyrene or glass provide sufficient adhesion to deposit stable filament surface patterns. After 3D-filament deposition the oil phase is floated off by the addition of aqueous medium.

As the two preconditions can be well fulfilled, a direct coupling of microfluidic and 3D-printing platforms is possible. We find that using a typical polymer stream volumetric flow rate of e.g. $Q=1000 \mu \mathrm{l} / \mathrm{h}$, 
construct volumes of $1 \mathrm{~cm}^{3} / \mathrm{h}$ can be printed with microtube inner diameters of $280 \mu \mathrm{m}$, strand widths of $200-500 \mu \mathrm{m}$, and printing velocities of $v_{\mathrm{p}}=5 \mathrm{~m} / \mathrm{s}$. This is in the range of commercial 3D-printing or bioprinting devices. (30) For typical microgel diameters of $100 \mu \mathrm{m}$ and cell numbers of 5 cells per microgel, cell densities of $10^{7}$ cells $/ \mathrm{cm}^{3}$ can potentially be achieved, which is suitable for tissue engineering applications. The direct coupling of microfluidics and 3D-printing enables continuous onestep construct fabrication from microgels, thereby removing the role of microfluidics as a typical fabrication bottleneck. The formulation of shear-thinning bioinks with appropriate elastic properites is not necessary, which also removes the issue of large bioprinting nozzle shear stresses with its negative effects on cell viability.

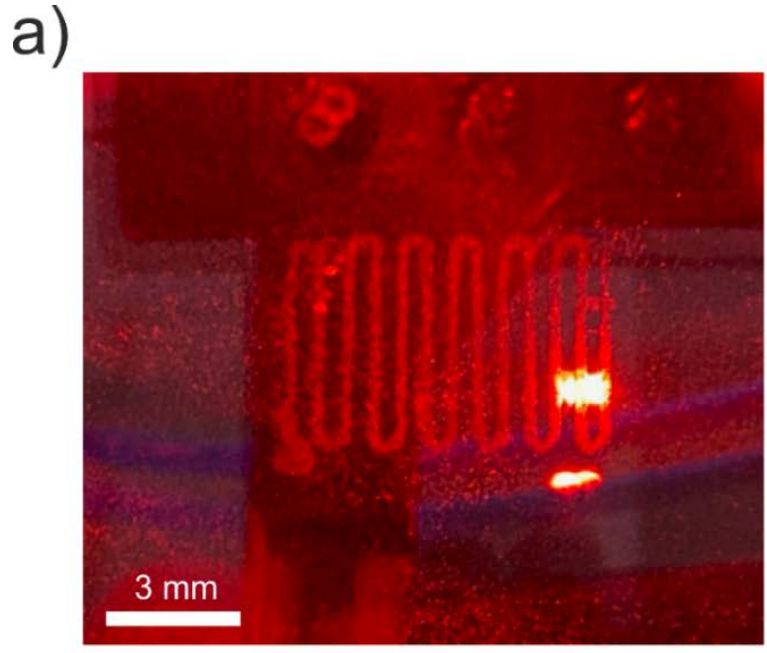

C)

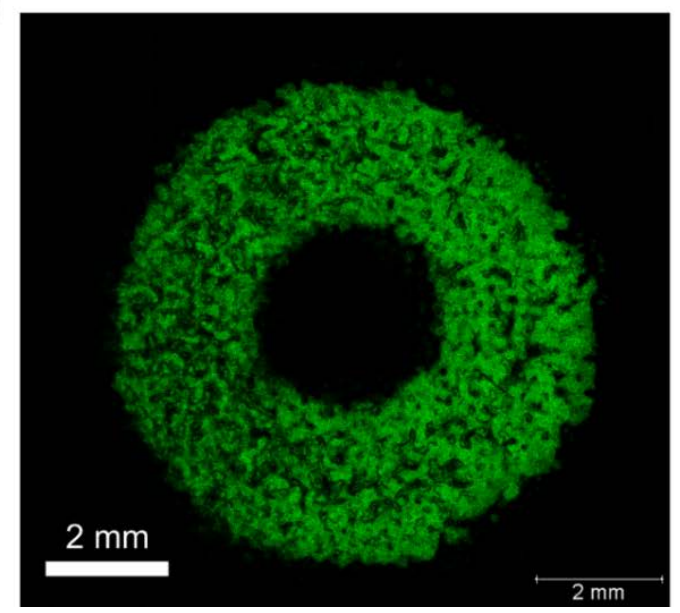

b)

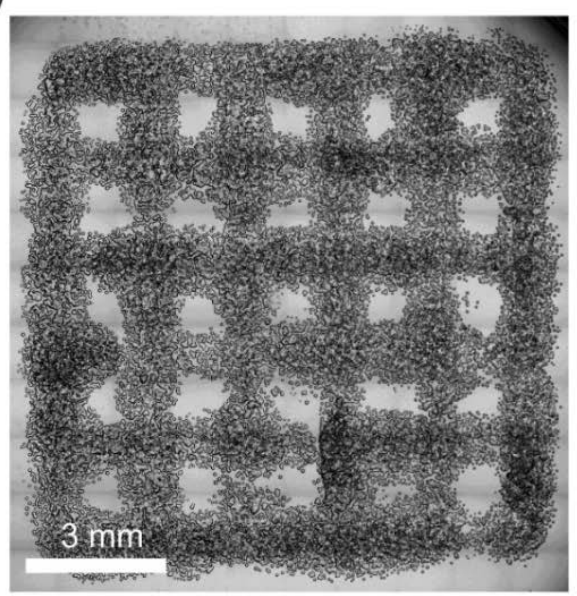

d)

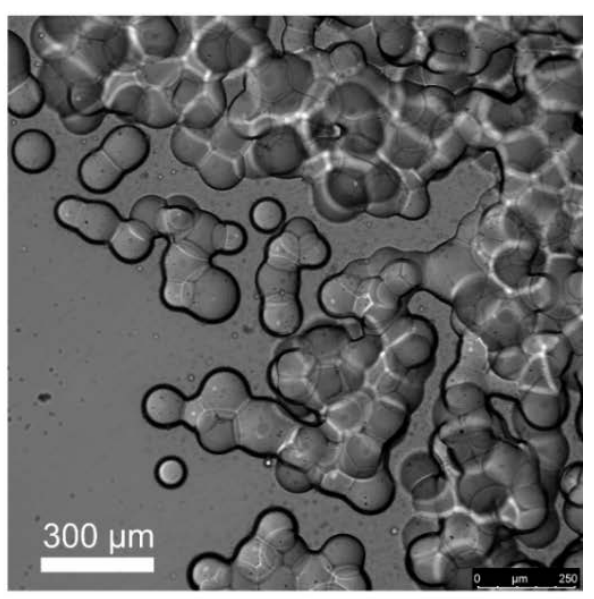

Fig. 3: a) Photograph of a standard meander-shaped layer fabricated by microgel filament deposition printing. The lines have a thickness of $300 \mu \mathrm{m}$. b) photograph of a cross-bar pattern obtained by on-top deposition of several microgel filaments. The average linewidth is $1 \mathrm{~mm}$. c) photograph of a donut-shaped microgel construct. The microgels have been fluorescently labelled by FITC-dextran to demonstrate the intrinsic microporosity corresponding to the black non-fluorescent regions, d) light microscopy image of a construct edge showing that fused adhesive microgels form a continuous, three-dimensional selfsupporting scaffold with intrinsic micropores. 
To assess the capabilities and limitations of the microfluidic printing process, we first investigated the printing of standard test patterns that are used for biofabrication. Fig. 3a shows a standard meander pattern where a line width of $300 \mu \mathrm{m}$ can be achieved. Fig. 3b shows a standard cross-bar pattern with line widths of $1 \mathrm{~mm}$. Each line consists of several overlayed filaments. We regularly observe that the first layer surface patterns can be printed with good precision and stability, whereas top layer patterns are less stable. We find that the contact adhesion (i) between adjacent microgels to form filaments, and (ii) between microgel filaments and substrate surfaces to form stable line patterns is sufficiently large, whereas (iii) the initial contact adhesion between subsequently printed filaments is relatively weak. Therefore, it was difficult to directly print three-dimensional constructs with large aspect ratio and overhangs, such as for 3D-crossbar patterns. Yet, 3D-objects with low aspect ratio such as the donut-shaped structure in Fig. 3c can readily be printed. In the future it should be possible to increase the filament contact adhesion by endowing the microgels with more rapidly acting physical or chemical adhesion groups.

Fig. 3d shows a magnification at the periphery of the donut shaped construct. Prolonged contact between microgel filaments leads to strong adhesion as apparent from the fused microgel structures, which form a three-dimensional percolating scaffold providing sufficient cohesion and mechanical stability for the construct. We observe that the initially weak contact adhesion between the microgel filaments after printing develops into a sufficiently strong adhesion to support high aspect structures over the course of minutes. We attribute the developing adhesion to chain interpenetration and entanglement formation at the periphery of the microgels, thereby mediating adhesion (tack) between microgels already at short contact times and low contact pressures. We find that if high aspect ratio structures are initially stabilized during the printing process, e.g. by external scaffolds or through rapidly acting physical interactions within the bioink, stable free-standing structures are obtained during the course of the printing process. The scaffolds can be removed directly afterwards.

A further feature observed in Figs. 3c and $d$ is the intrinsic microporosity of the microgel constructs. As the printed microgel filaments initially have limited contact adhesion, they show variable displacements from the line patterns. This leads to void structures, whose extension, diameter and volume depend on the printed line density. These voids provide microgel constructs with an intrinsic microporosity as seen in the donut shaped construct in Fig. 3c. Typical voids are in the form of channels with widths of the order of the microgel diameters, i.e. 50 - $200 \mu \mathrm{m}$. Depending on the line density, microchannel lengths can be of the order of a few hundred microns up to the dimension of the construct. The intrinsic microporosity of microgel constructs is important for perfusion to provide cells with nutrients. Culture media can perfuse through the micropore channels at much higher velocity compared to solid homogeneous hydrogel 
constructs. Thus, for microgel constructs the integration of three-dimensional micro vascular structures is not necessary for the provision of nutrients or drugs to encapsulated cells.

We find the currently achievable line width of microgel filament deposition to be in the range of 200 $500 \mu \mathrm{m}$. In principle, for ideal pearl-necklace filaments that could be positioned with sufficient precision, the achievable line widths would be equal to the microgel diameters, i.e. in the range of $50-200 \mu \mathrm{m}$. In practice we needed to let the microgels jam into sligthly thicker filaments with cross-sections containing typically 2-5 microgels. This thickness is a compromise between a printhead velocity to be as large as possible to draw thin microgel filaments, but not too large to avoid filament rupture. If the contact adhesion between microgels and microgels filaments could be further increased, the direct fabrication of high-aspect ratio structures with down to $100 \mu \mathrm{m}$ feature size should well be possible. Yet, with the temporal stabilization of the constructs by scaffolds during the printing process, a direct fabrication of three-dimensional functional microgel constructs is already possible as will be subsequently demonstrated.

\section{Direct in-flow 3D printing of permeable constructs}

In Fig. 4 we show the direct filament printing of free-standing 3D constructs into a perfusion chamber by using stabilizing scaffolds, which can be removed after printing. Fig. 4a shows a scheme of the perfusion chamber having in-flow and out-flow ports $\mathrm{C}_{\mathrm{in}}$ and $\mathrm{C}_{\text {out }}$ connecting to an upstream and downstream chamber, which contain the scaffolds. With the design in Fig. 4a simple rectangular box scaffolds can be printed, which seals to the side walls of the perfusion chamber. Other scaffold shapes are possible to produce microgel constructs of variable shapes. 
a) $\begin{gathered}\text { usstrean } \\ \text { chamber }\end{gathered}$
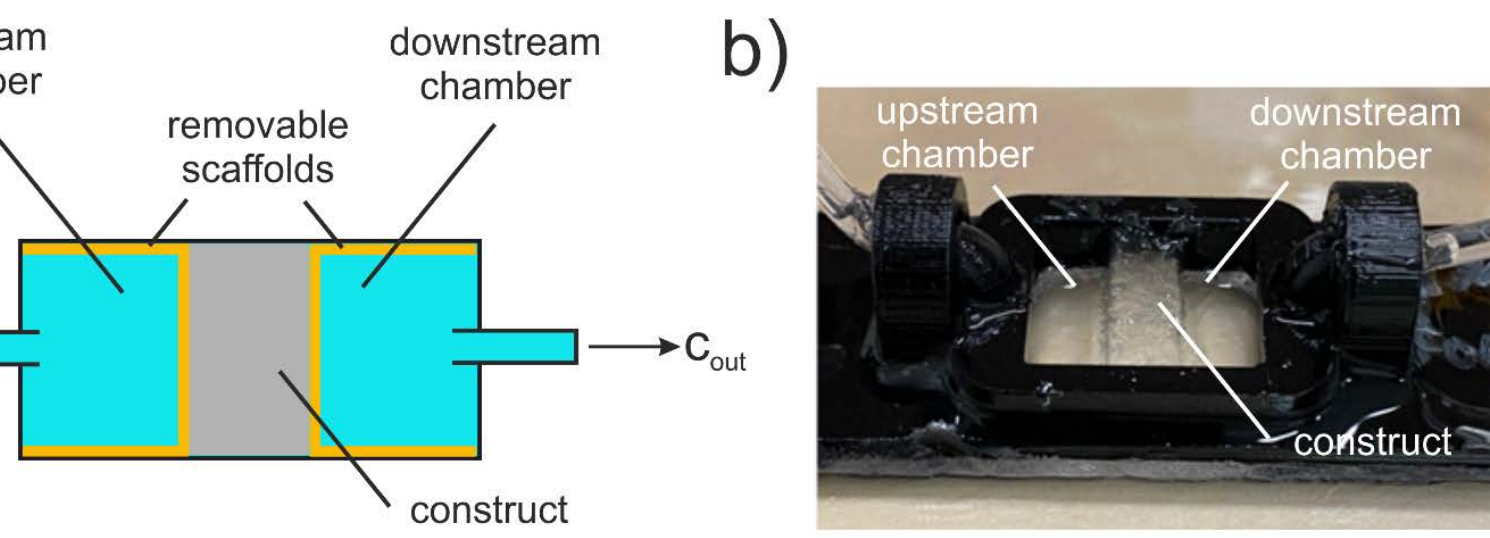

c)

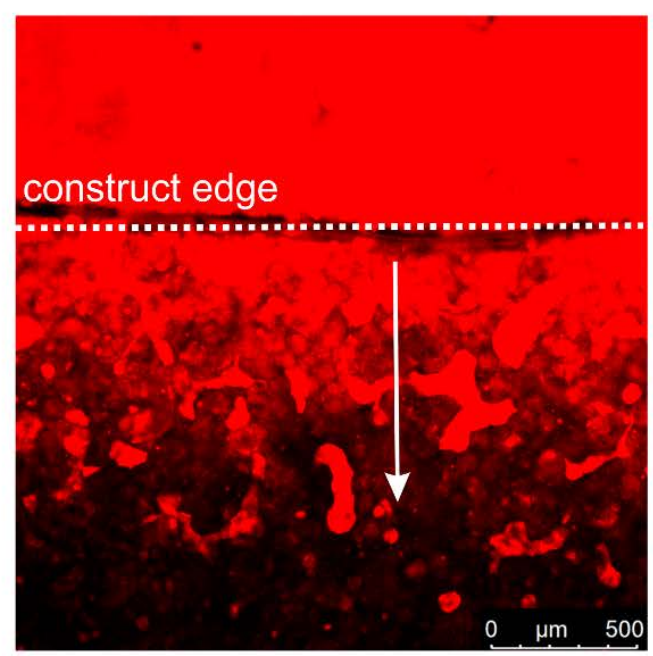

d)

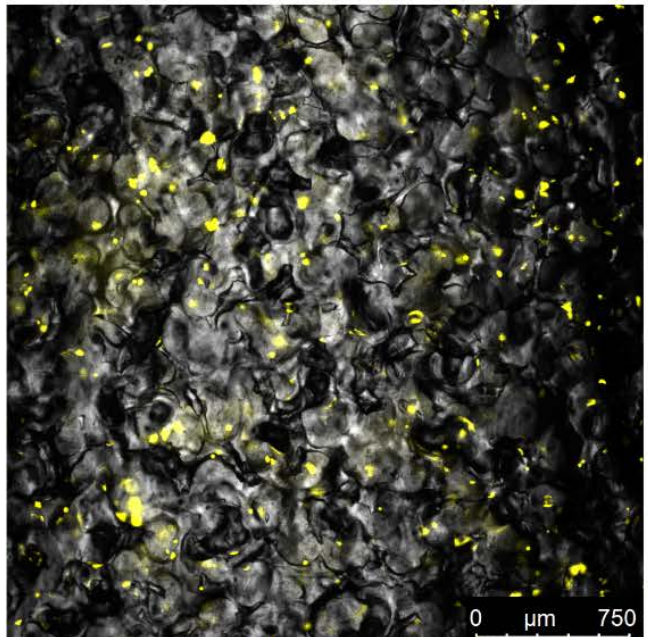

Fig. 4: a) Scheme of the perfusion chamber consisting of an upstream and downstream chamber, perfusion ports, and removable scaffolds to stabilize the microgel construct during 3D-printing, b) photograph of a microgel construct in the perfusion chamber directly after printing and removal of the scaffolds, c) confocal microscopy image of the permeation front of a fluorescent dye, where the high dye concentration in the micropores can be clearly seen, d) confocal microscopy image of YFP-labelled HEK-cells within a microgel construct.

The perfusion chamber has been specifically designed to provide stable media perfusion for long-term culture, and to be compatible with optical and fluorescence microscopy for live cell imaging. The chamber is fabricated from an FDM-printed PLA-housing that is glued onto a thin petri dish polystyrene base plate having the dimension as a standard microscopy slide. The chamber can be connected via luer lock inserts to external tubings. The perfusion chamber with the integrated construct can be used as an open device to manually add solutions and reagents, or sealed with a thin cover slide to obtain a closed system. The temperature is controlled by mounting on a Peltier stage. The permeation chamber allows to provide the construct with fresh medium, and to perform construct perfusion experiments with controllable flow rates. 
During printing, the oil phase vertically separates from the construct. As the oil has a lower density compared to aqueous solutions and the microgels, it instantaneously phase separates and floats on top of the printed construct. Eventually the oil phase floats over the scaffolds into the upstream and downstream permeation chambers and can be removed by either by automated pumping or by manual pipetting from the perfusion chamber.

Scaffold removal can be done immediately after printing for reusable scaffolds, or by subsequent dissolution in the aqueous medium for sacrificial scaffolds. We observe that microgel filament adhesion is sufficiently strong to provide cohesion and thus three-dimensional mechanical stability of the printed constructs in water and in air. This is shown in Fig. 4b, where the scaffolds have been removed from a 10 x $5 \times 3 \mathrm{~mm}^{3}$ (width $\mathrm{x}$ depth $\mathrm{x}$ height) construct directly after printing. Here, the construct is printed to be in contact with the perfusion chamber side walls for sealing and subsequent perfusion experiments. Examples of completely free-standing 3D-constructs are shown in the Supporting Information. We find that the microgel constructs well preserves the shape of the scaffold and are long time stable as shown in the Supporting Information (Figs. S19, S20).

We observe that the intrinsic microporosity of the microgel constructs provides complete permeation already at hydrostatic pressures of a few millimeters, which can be realized by filling aqueous solutions into the upstream chamber. The confocal microscopy image in Fig. 4c shows the hydrostatic pressuredriven permeation of a solution of a fluorescent dye (ATTO 647N) through the construct via the micropores. We observe that the fluorescent solution front advances via advection through the micropore channels, together with diffusion through the microgels.

As examples for cell encapsulation we chose yeast cells (saccharomyces cerevisiae) for optimization of the method, and YFP-tagged HEK293T-cells for the final device tests. The confocal microscopy image in Fig. 4d shows a homogeneous distribution of the yellow fluorescent HEK cells in the final microgel construct matrix, imaged in the perfusion chamber.

When printed into the perfusion chamber the constructs can be fabricated to make direct contact and sealing with the side walls, and direct contact with the top cover slide to allow continuous microperfusion of the whole construct. This configuration can be used for applications involving cell tests for drugs, or as bioreactors where production cells in the constructs convert precursors to molecular or macromolecular drugs that are released into the downstream chamber for continuous production. 


\section{Integration of complementary perfusion channels}

a)
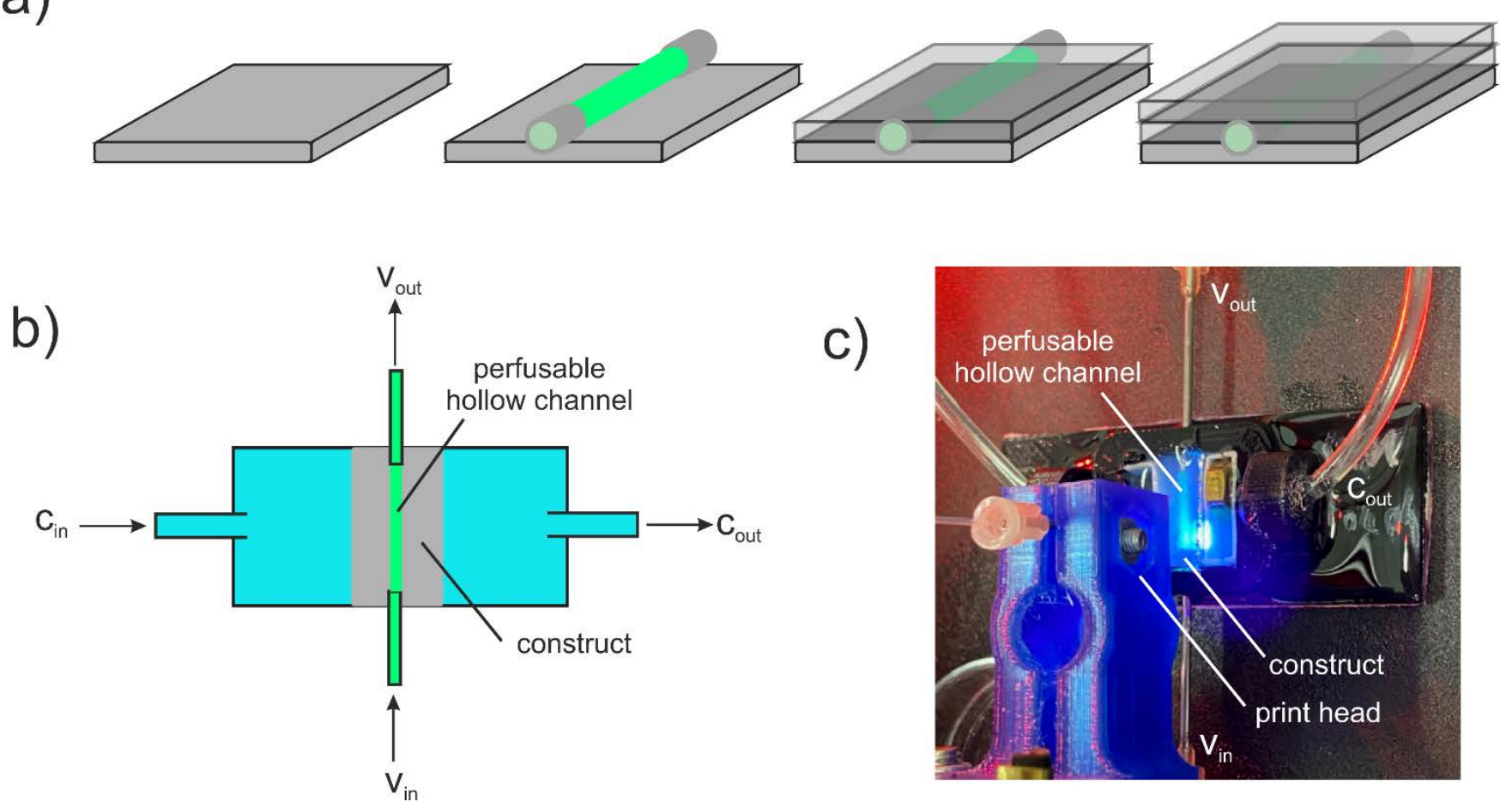

c)

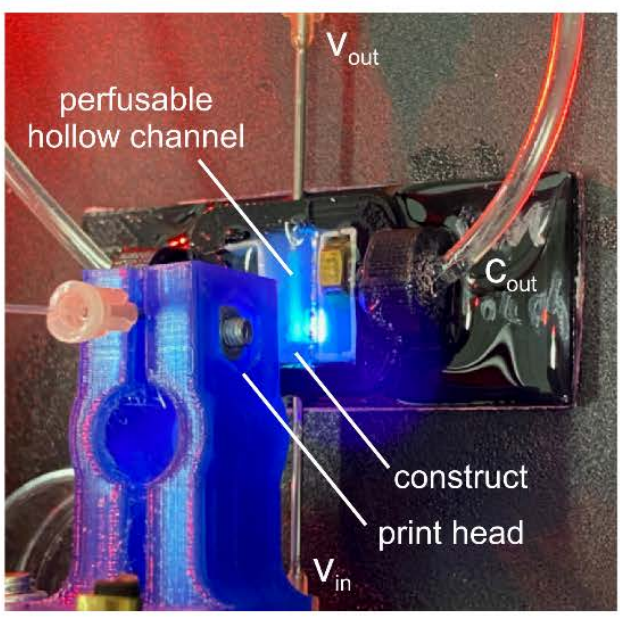

Fig. 5: a) Layer-by-layer printing of microgel construct with integrated perfusion channel. After printing of the first layer, a hollow perfusion channel is inserted. Subsequently, the second and third layers are printed. b) The construct is directly printed into a perfusion chamber. The perfusion chamber provides whole construct permeation via flows $C_{i n}$ and $c_{o u t}$, as well as independent flow through the perfusion channel via flows $v_{\text {in }}$ and $v_{\text {out. }}$. C) Photograph of a perfusion chamber containing the construct directly after printing. The flow of the fluorescein solution through the integrated PVA hollow channel is clearly visible.

Because of the yet limited filament contact adhesion the direct printing of larger perfusion channels is still difficult. To fabricate 3D-constructs with integrated perfusable microchannels we follow the same printing method as described above, employing a scaffold that can be removed after printing. The integration of the microchannel proceeds via a layer-by-layer procedure as schematically shown in Fig. 5a. After the first layer is printed, a hollow PVA microtube is inserted on top of the layer. The microtubes are fabricated by 3D-FDM printing from commercial polyvinylalcohol (PVA) filaments with $0.4 \mathrm{~mm}$ inner diameter and $0.9 \mathrm{~mm}$ outer diameter. PVA dissolves well in water, is an FDA-approved polymer, and has excellent FDM-printing properties. We found that a wall thickness of $1 \mathrm{~mm}$ provides sufficient integrity of the PVA microtube during perfusion over time periods of several hours, before it dissolves. To provide sufficient microchannel integrity after PVA-dissolution, we coated the PVA microtubes with a photo-crosslinked polyoxazoline layer using the same photochemistry as for the polyoxazoline microgel fabrication. The microtube inlet and outlet were equipped with short PDMS microtubing connections. These serve to 
directly connect the microtube to the inlet and outlet perfusion needles of the perfusion chamber directly after insertion of the microchannel, as shown in Fig. 5c.

After integration of the microchannel, microgel filaments are printed around the microtube to finish the second layer. Subsequently, a third layer is printed on top of the second layer to finish the construct. The microchannel is already functional after its integration and can be perfused during the subsequent construct printing process. This is shown in Fig. 5c, where a fluorescein solution that is perfused through the microchannel can be well observed. Typical flow rates in the microchannel are 1-2 ml/h. At an inner diameter of $500 \mu \mathrm{m}$ this corresponds to flow velocities of $1-2 \mathrm{~mm} / \mathrm{s}$ which is in a typical range for blood capillaries in view of later applications.

The integration of the complementary perfusion channel system allows to microperfuse the construct as a whole via a flow $c_{\text {in }}$ from the upstream chamber through the microporous construct, and then exiting via the downstream chamber as $\mathrm{C}_{\text {out. }}$ In addition, the construct can be separately perfused via a flow $\mathrm{V}_{\text {in }}$ from the inlet port through the microchannel system, exiting downstream via the outlet port vout. The separate channel systems allows infusion with endothelial cells (e.g. HUVECs) for vascularization, and a spatially defined provision of nutrients or factors to the cells in the construct. Besides the simple straight channel shown in Fig. 6, also multiple channels with bifurcation points can, in principle, be integrated into the construct and connected to the perfusion chamber.
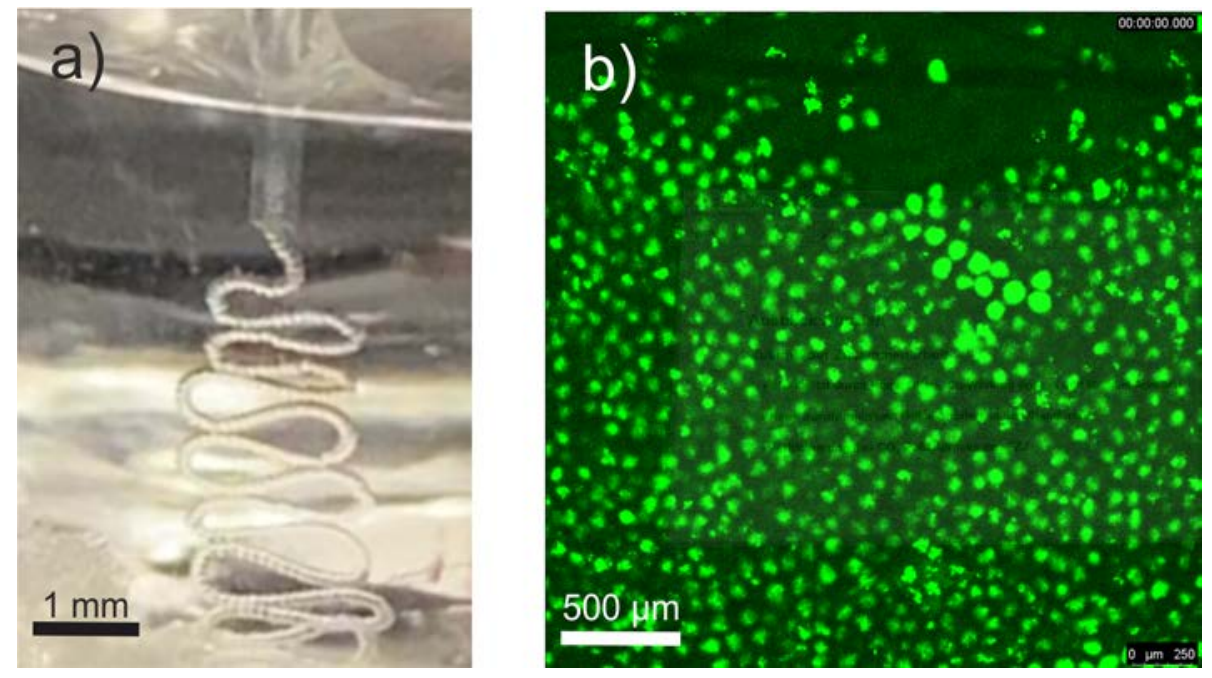

Fig. 6: a) Photograph of an alginate capsule fiber formed after exiting the microtube. b) Confocal fluorescence microscopy image of part of a 3D-printed alginate capsule construct. The fluorescence arises from encapsulated fluorescently labelled polystyrene microbeads to demonstrate the integrity and stability of the alginate capsules. 


\section{In-flow bioprinting of alginate microcapsule constructs}

So far the method had been established for polyoxazoline microgels, which can be well fabricated in microfluidic devices by fast photo-crosslinking. To extend the method from synthetic polymers with chemical cross-linking to biopolymers with physical cross-linking, we considered the fabrication of alginate microgel constructs. Therefore, an alginate solution was focused with an oil stream (1-undecanol) into the nozzle to generate alginate solution droplets. For rapid on-chip crosslinking calcium ethylhexanoate was dissolved in the oil-phase. We find that a continuous production of alginate microcapsules at high flow rates is possible.

When exiting the microtubing, alginate capsules form very stable pearl-necklace filaments as shown in Fig. 6a. These can be printed into alginate microcapsule constructs similarly as demonstrated for the polyoxazoline microgel constructs. Fig. 6b shows a confocal microscopy image of the microcapsule construct. Within the construct the microcapsules have a deformed and folded outer shape, but an intact capsule wall as demonstrated by the fluorescein-labelled polystyrene beads that stay inside the capsules.

\section{Direct coupling between microfluidics and 3D-printing}

The aim of this study was the integration of microfluidics and 3D-printing to fabricate functional microgel constructs in one continuous process. The first prerequisite to directly connect both methodologies is the compatibility of the microgel flow velocity in the microfluidic system with the print head speed of the 3D printer. The second prerequisite is sufficient stability of the microgel filaments for 3D-printing. We find the first device-related condition to be well fulfilled for standard operation conditions for both methodologies. The fulfillment of the second condition is a surprising finding of this study. The observation of stable microgel filament formation can be rationalized by the reduction of the oil/water surface area leading to jamming, together with a sufficient adhesion when the microgels are jammed into contact. Microgels are characterized by moderate cross-linking densities and high polymer chain mobility at their periphery (31), such that chain interpenetration and entanglement formation can mediate adhesion of microgels already at short contact times and low contact pressures. High polymer segment mobility, e.g. well above the glass-transition temperature, together with solvent swelling to facilitate entanglement formation are known properties promoting fast contact adhesion, known as tack, e.g. in polymer adhesives. (32)

Thus the direct coupling of both methodologies for the fabrication of microgel constructs is straightforward, and a separate formulation-step of a bioink is not needed. An issue to be further addressed 
is an improved filament contact adhesion for the direct fabrication of free-standing high aspect ratio constructs, which could be approached externally by temporary scaffold structures or internally by endowing the microgels with more rapidly acting physical or chemical adhesion groups.

The special microfluidic channel design allows 3D-focusing to localize the cells in the center stream to minimize shear stress. Double focusing allows cell-prefocusing and on-chip addition of cross-linking agents. The 3D orifice design enables to access all relevant hydrodynamic regimes for a controlled formation of a wide range of droplet diameters, where the droplet size can be precisely controlled by the Capillary number Ca. The post-chip fast in-flow photo-crosslinking step continuously generates monodisperse, cell-laden microgels.

Besides microfluidics and 3D-printing, a further aspect was the direct integration of the printed construct into a perfusion chamber suitable for long-term live cell imaging, which can be used as a bioreactor, for cell testing and for tissue regeneration. Therefore we realized a one-step continuous production process based on microfluidics and 3D-printing to directly fabricate cell-laden permeable constructs in functional perfusion chambers for immediate use in cell studies. Photographs of the complete setup are shown in the Supporting Information (Figs. S23, S24). The process can in principle be automated to print series of construct/perfusion chamber designs. The direct coupling of the methods removes the role of microfluidics as a bottleneck in biofabrication processes.

\section{Structural hierarchies}

The described method allows to control the structural hierarchy of biofabricated constructs on three hierarchy levels. On the first level within individual microgels, i.e. on length scales below $\sim 100 \mu \mathrm{m}$, the local cell environment (pericellular environment) can be adjusted via the control of

- the microgel diameter via the droplet size and capillary number as shown in Fig. 3,

- the number and types of encapsulated cells via the cell concentrations in the initial solutions and the flow rate ratios in the central and first focusing streams,

- the local viscoelastic properties of the polymer matrix via the degree of functionalization and molecular weight of the precursor polymer, the concentration of the photoinitiator, and the light intensity,

- the local (bio)chemical functionality via specific functionality of the polymer,

- the internal structure from homogeneous structures as for the POx-microgels, to core/shellstructures as for the alginate capsules. 
The control of the cell number is possible within the limits of the Poisson distribution, whereas all other parameters can be controlled with good accuracy. The microgel diameter controls the diffusion times of agents to the cells. The microgels thus represent micro compartments where the cells are located in a biochemically and mechanically tailored microenvironment.

The second hierarchy level concerns the level of the filaments and micropores within the construct, i.e. on length scales of $100-500 \mu \mathrm{m}$. The average inter filament distance can be controlled by the line distance during 3D-printing, which sets the diameter, volume and degree of percolation of the micropore channel system. The micropore system allows the sustainable permeation with buffer solutions or culture medium potentially also for larger constructs.

Furthermore, similar as for homogeneous hydrogel constructs, a third hierarchy level on length scales from $>500 \mu \mathrm{m}$ to the size of the total construct can be realized, where multilayers, integrated microchannels, and specific construct shapes can be fabricated and designed. The direct printing and thereby integration into a specially designed perfusion chamber allows whole construct permeation via the intrinsic microchannels, as well as complementary localized perfusion through the microchannel system with separate in- and outflow connections. In principle, by adjusting the flow rates of different

cell, nutrient and growth factor streams in the microfluidic entrance channels $A, B_{1}$, and $B_{2}$ during 3D printing, construct regions with specific cell types can be realized to mimic native tissue architecture. Therefore, on the level of the construct within the permeation chamber, flows of nutrients and drugs can be controlled for live cell studies.

\section{Conclusions}

The aim of the present work was the integration of microfluidic droplet generation, cell encapsulation, inflow photo crosslinking, and 3D-printing in one continuous process to directly print cell-laden microgels into three-dimensional constructs in flow chambers. We showed that the two preconditions, i.e. microgel flow rate being equal to the print head speed, and the formation of printable microgel filaments, can be fulfilled with standard microfluidic and 3D-printing devices using conventional microgel materials. This allows one to establish an automatable process, where cell-laden microgels are continuously fabricated and printed into perfusable scaffolds. As these scaffolds are integrated into flow chambers for long-term live cell imaging, they can be used as a bioreactor, for cell testing and tissue regeneration. A further advantage of microgel scaffolds is their intrinsic microporosity, which provides a perfusion/diffusionpathway with transport rates on the order of centimeters per hour to support a sustained provision of nutrients, factors and agents to the cells in thick constructs. The integration of and connection to hollow 
perfusion channel inserts was demonstrated in view of the realization of vascular channel systems. The perfusion chamber allows for continuous perfusion of the whole construct via its microporosity, and for separate perfusion through perfusion channel inserts. For microfluidic channel system a double 3Dfocusing design was demonstrated to minimize cell shear stress during encapsulation, to access a large range of microgel diameters, and to allow premixing with cells, crosslinking reagents, medium and factors. The method is demonstrated for photo-chemically cross-linked polyoxazolines as well as physically cross-linked alginate microgels for yeast and human embryonic kidney cells. We therefore resolve the current microfluidic production bottleneck of microgels for bioinks through the design of an integral and continuous automatable fabrication process from microgel production to bioprinting that can even be integrated in live cell perfusion chambers.

\section{Supporting Information}

Supporting Information is available from the Wiley Online Library or from the author.

\section{Acknowledgements}

Funded by the Deutsche Forschungsgemeinschaft (DFG, German Research Foundation) - Projektnummer 326998133 - TRR 225 (subproject A06).

\section{Conflicts of interest}

The authors declare no conflicts of interest.

\section{Data availability}

All of this study are available on request.

\section{Keywords}

biomaterials, microgels, microfluidics, 3D printing, bioprinting 


\section{References}

1. A. Atala, Chem. Rev. 2020, 120, 10545-10546.

2. J. Groll, J. A. Burdick, D. W. Cho, B. Derby, M. Gelinsky, S. C. Heilshorn, T. Jüngst, J. Malda, V. A Mironov, K. Nakayama, A. Ovisanikov, W. Sun, S. Takeuchi, J. J. Yoo, T. B. F. Woodfield, Biofabrication 2019, 11, 013001.

3. W. Sun, B. Starly, A. C. Daly, J. A. Burdick, J. Groll, G. Skeldon, W. Shu, Y. Sakai, M. Shinohara, M. Nishikawa, J. Jang, D.-W. Cho, M. Nie, S. Takeuchi, S. Ostrovidov, A. Khademhosseini, R. D. Kamm, V. Mironov, L. Moroni, I. T. Ozbolat, Biofabrication 2020, 12, 022002.

4. R. Levato, T. Juengst, R. G. Scheuring, T. Blunk, J. Groll, J. Malda, Adv. Mater. 2020, 32, 1906423.

5. C. B. Highley, K. H. Song, A. C. Daly, J. A. Burdick, Adv. Sci. 2019, 6, 1801076.

6. D. Velasco, E. Tumarkin, E. Kumacheva, Small 2012, 8, 1633-1642.

7. W. Jiang, M. Li, Z. Chen, K. W. Leong, Lab Chip 2016, 16, 4482-4506.

8. A. C. Daly, L. Riley, T. Segura, J. A. Burdick, Nat. Rev. 2020, 5, 20-43.

9. A. S. Mao, B. Özkale, N. J. Shah, K. H. Vining, T. Descombes, L. Zhang, C. M. Tringides, S.-W. Wong, J.-W. Shin, D. T. Scadden, D. A. Weitz, D. J. Mooney, Proc. Natl. Acad. Sci. 2019, 116, 1539215397.

10. S. R. Pajoumshariati, M. Azizi, D. Wesner, P. G. Miller, M. L. Shuler, A. Abbaspourrad, ACS Appl. Mater. Interfaces 2018, 10, 9235-9246.

11. A. S. Mao, J.-W. Shin, S. Utech, H. Wang, O. Uzun, W. Li, M. Cooper, Y. Hu, L. Zhang, D. A. Weitz, D. J. Mooney, Nat. Mater. 2017, 16, 236-243.

12. P. S. Lienemann, T. Rossow, A. S. Mao, Q. Vallmajo-Martin, M. Ehrbar, D. J. Mooney, Lab Chip, 2017, 17, 727.

13. F. Chen, J. Xue, J. Zhang, M. Bai, X. Yu, X.; C. Fan, Y. Zhao, J. Am. Chem. Soc. 2020, 142, 28892896.

14. Q. Feng, Q. Li, H. Wen, J. Chen, M. Liang, H. Huang, D. Lan, H. Dong, X. Cao, Adv. Funct. Mater., 2019, 29, 1096690. 
15. L. P. B. Guerzoni, T. Yoshinari, D. B. Gehlen, D. Rommel, T. Haraszti, M. Akashi, L. De Laporte, Biomacromolecules 2019, 20, 3746-3754

16. T. Rossow, J. A. Heyman, A. J. Ehrlicher, A. Langhoff, D. A. Weitz, R. Haag, S. Seiffert, J. Am. Chem. Soc. 2012, 134, 4983-4989.

17. E. Kapourani, F. Neumann, K. Achazi, J. Dernedde, R. Haag, Macromol. Bioscience 2018, 18, 1800116

18. H. Wang, H. Liu, H. Liu, W. Su, W. Chen, J. Qin, Adv. Mater. Technol. 2019, 4, 1800632.

19. C. Fan, S.-H. Zhan, Z.-X. Dong, W. Yang, W.-S. Deng, X. Liu, P. Suna, D.-A. Wang, Mater. Sci. Eng. C 2019, 108, 110399.

20. A. M. Compaan, K. Song, W. Chai, Y. Huang, ACS Appl. Mater. Interfaces 2020, 12, 7855-7868.

21. S. L. Anna, H. C. Mayer, Phys. Fluids 2006, 18, 121512.

22. T. Ward, M. Faivre, M. Abkarian, H. A. Stone, Electrophoresis 2005, 26, 3716-3724.

23. F. Lapierre, N. Wu, Y. Zhu, Proc. SPIE 2011, 8204, 82040H-1.

24. C. A. Stan, S. K. Y. Tang, G. M. Whitesides, Anal. Chem. 2009, 81, 2399-2402.

25. J. Tan, J. H. Xu, S. W. Li, G. S. Luo, Chem. Eng. J. 2008, 136, 306-311.

26. R.-C. Luo, C.-H. Chen, Soft 2012, 1, 1-23.

27. C. H. Choi, J. H. Jung, T. S. Hwang, C. S. Lee, Macromol. Res. 2009, 17, 163-167.

28. A. J. D. Krüger, O. Bakirman, P. B. Guerzoni, A. Jans, D. B. Gehlen, D. Rommel, T. Haraszti, A. J. C. Kuehne, L. De Laporte, Adv. Mater. 2019, 31, 1903668.

29. D. B. Kolesky, K. A. Homan, M. A. Skylar-Scott, J. A. Lewis, Proc. Natl. Acad. Sci. 2016, 113, $3179-3184$

30. A. K. Miri, I. Mirzaee, S. Hassan, S. M. Oskui, D. Nieto, A. Khademhosseini, Y. S. Zhang, Lab Chip 2019, 19, 2019.

31. F. A. Plamper, W. Richtering Acc. Chem. Res. 2017, 50, 131-140.

32. S. Sun, M. Li, A. Liu, Int. J. Adhesion Adhesives 2013, 41, 98-106. 


\section{Acknowledgements}

Funded by the Deutsche Forschungsgemeinschaft (DFG, German Research Foundation) - Projektnummer 326998133 - TRR 225 (subproject A06)

\section{Competing interests}

The authors declare no competing interests.

\section{Additional information}

Supplementary Information The online version contains supplementary material available at https:// 